\title{
A Realidade Acerca da Vitamina D numa População Adolescente
}

\section{The Reality about Vitamin D in an Adolescent Population}

Hugo de Castro Faria, Sofia Carneiro ${ }^{2}$, Carla Santos ${ }^{1}$, Cláudia Cristovão1, Ana Serrão Neto ${ }^{1}$

\section{RESUMO}

INTRODUÇÃO: A vitamina D é essencial para a formação óssea e a fase da adolescência é fundamental para garantir saúde óssea ao longo da vida adulta. Têm sido associadas diversas outras patologias a níveis de vitamina D subótimos. Diversos estudos em redor do globo têm mostrado prevalência de défice e insuficiência de vitamina D muito elevados, contudo persistem dúvidas acerca de quais os valores de cut-off que deverão ser utilizadas ao avaliar os seus níveis séricos.

METODOLOGIA: Com este estudo pretendemos avaliar os níveis de 25-hidroxivitamina D numa população adolescente e relacionar estes valores com diversas variáveis relevantes: caracterização demográfica, dados antropométricos, fotótipo, estádio de desenvolvimento pubertário, variáveis relacionadas com hábitos de vida que podem interferir com status da vitamina D nomeadamente, tipo de dieta, exposição solar, utilização de protetor solar, utilização de chapéu, meio de transporte de e para escola, prática desportiva e doenças associadas.

RESULTADOS: Obtivemos uma amostra de 320 adolescentes, os valores de vitamina D variaram entre 6,4 e 61,4 ng/mL, com média de 26,4 \pm 8,5 ng/mL e foram considerados ótimos em 30,3\% dos doseamentos, insuficientes em 49,1\% e deficientes em 20,6\% dos doseamentos.

CONCLUSÃO: Conseguimos demonstrar que sexo feminino e os fotótipos IV e $V$ são fatores de risco para valores inferiores ao recomendado e a prática de atividade física ao ar livre é fator protetor. A elevada prevalência de insuficiência e deficiência, vem reforçar a necessidade de aprofundar estudos acerca do cut-off ideal para esta vitamina.

PALAVRAS-CHAVE: Adolescente; Deficiência de Vitamina D; Vitamina D 


\section{ABSTRACT}

INTRODUCTION: Vitamin D is essential for bone formation and the adolescence period is essential to ensure bone health throughout adult life. Several other pathologies have been associated with suboptimal vitamin D levels. Several studies around the globe have shown a very high prevalence of vitamin D deficiency and insufficiency, however doubts remain about which cut-off values should be used when assessing their serum levels.

METHODOLOGY: With this study we intend to evaluate the levels of 25-hydroxyvitamin $D$ in an adolescent population and relate these values with several relevant variables: demographic characterization, anthropometric data, phototype, pubertal development stage, variables related to lifestyle habits that can interfere with status vitamin D, namely, type of diet, sun exposure, use of sunscreen, use of hat, means of transport to and from school, sports and associated diseases.

RESULTS: We obtained a sample of 320 adolescents, vitamin D values varied between 6.4 and $61.4 \mathrm{ng} / \mathrm{mL}$, with an average of $26.4 \pm 8.5 \mathrm{ng} / \mathrm{mL}$ and were considered excellent in 30.3\% of the patients, dosages, insufficient in $49.1 \%$ and deficient in $20.6 \%$ of the dosages.

CONCLUSION: We were able to demonstrate that female gender and phototypes IV and $\mathrm{V}$ are risk factors for values lower than recommended and the practice of physical activity outdoors is a protective factor. The high prevalence of insufficiency and deficiency reinforces the need for further studies on the ideal cut-off for this vitamin.

KEYWORDS: Adolescent; Vitamin D Deficiency; Vitamin D

\section{INTRODUÇÃO}

A vitamina D é um nutriente essencial cujo papel na saúde óssea e homeostasia do cálcio é de extrema importância.

A associação entre a deficiência grave de vitamina D e doença é bem conhecida, nomeadamente o raquitismo em lactentes e crianças pequenas e osteomalácia ou hipocalcémia com consequente risco de tetania ou convulsões em todas as idades. ${ }^{1-5}$

Estas complicações são associadas sobretudo a populações desnutridas por défice de recursos.

Recentemente tem ganho relevância a preocupação com défices menos severos de vitamina $D$, não sendo clara a relevância ou significado clínico da deficiência ligeira ou moderada. Diversos estudos têm revelado elevada prevalência de valores subótimos de vitamina D em todo o mundo, sobretudo entre consumidores de "fast food", pessoas de pele escura, obesos, pessoas com baixa exposição solar ou utilizadores de protetor solar e em localizações afastadas da linha do equador. ${ }^{6}$ A obesidade, terapêutica com anti-convulsivantes, glucocorticoides e antirretrovirais são também fatores de risco descritos. ${ }^{7}$

Evidência recente sugere que deficiência subclínica em vitamina D, durante a adolescência, pode afetar a formação óssea, reduzindo o pico de massa óssea e predispondo para osteoporose na idade adulta. Suplementação com esta vitamina em adolescentes com défice pode melhorar a sua densidade óssea. ${ }^{8-10}$

A principal fonte de vitamina D é a síntese cutânea através de um processo iniciado pela radiação solar. A utilização de protetor solar impede este processo. ${ }^{11}$ Esta capacidade de produzir vitamina D varia com diversos fatores, nomeadamente, o tipo de pele, a altura do ano e a localização geográfica. ${ }^{12}$ A dieta apenas consegue fornecer pequenas quantidades desta vitamina essencial, sendo as principais fontes os peixes ricos em gordura e o ovo. Existem atualmente diversos alimentos fortificados com vitamina D, mas esta vitamina é lipossolúvel, o que coloca dificuldades na fortificação em quantidades elevadas.

Existe atualmente intenso debate acerca do valor sérico de 25-hidroxivitamina D $(25(\mathrm{OH}) \mathrm{D})$ que traduz um adequado status vitamínico, mas estudos relevantes referem que valores superiores a 32 ng/mL são necessários para garantir uma absorção de cálcio eficiente. ${ }^{13}$ Para atingir este status seria necessário uma ingestão diária de cerca de 1063 IU. Vários estudos têm demonstrado elevada prevalência de insuficiência de vitamina D em sociedades semelhantes à portuguesa. Nos Estados Unidos, a prevalência de deficiência e insuficiência nesta vitamina é aproximadamente 15\%, em idade pediátrica. ${ }^{14-16}$ Em Portugal há poucos estudos em adolescentes. Dois estudos portugueses recentes, com adolescentes, revelaram concentrações médias de $25(\mathrm{OH}) \mathrm{D}$ de cerca de $16,5 \mathrm{ng} / \mathrm{mL} .{ }^{17,18}$ 
O significado clínico destes valores e suas consequências são ainda incertos. Estudos epidemiológicos têm sugerido associação a doenças autoimunes como esclerose múltipla, ${ }^{19}$ diabetes tipo $1,{ }^{20}$ artrite reumatoide, ${ }^{21}$ doença inflamatória intestinal,22 alterações do humor, ${ }^{23,24}$ doença cardiovascular ${ }^{18,25}$ e doenças oncológicas como cancro do pâncreas, mama e cólon. ${ }^{26-30}$ Contudo, a relação causal destas associações bem como o seu mecanismo não são conhecidos.

Existe divergência na literatura e entre recomendações sobre qual o valor ideal desta vitamina. A Society for Adolescente Health and Medicine (SAHM) define ${ }^{31}$ deficiência valores de 25(OH)D inferiores a $20 \mathrm{ng} / \mathrm{mL}$ e valores ótimos acima de $30 \mathrm{ng} / \mathrm{mL}$, mas outras organizações como a Pediatric Endocrine Society recomendam que estes limiares se situem em 12 e 20 ng/mL respetivamente. ${ }^{32}$ As primeiras recomendações baseiam-se em estudos que relacionam níveis de $25(\mathrm{OH})$ D com níveis de hormona paratiroideia, a absorção de cálcio e densidade óssea, enquanto as segundas se baseiam em estudos radiológicos da densidade óssea e no facto de haver baixa evidência em crianças que valores superiores a 20 ng/mL sejam necessários para otimizar absorção do cálcio e densidade óssea, embora existam estudos em adultos que mostram esta necessidade. Alguns autores referem que valores superiores a 30 ng/mL são necessários para garantir as ações extra-esqueléticas. ${ }^{33}$

A SAHM recomenda suplementação com vitamina D a todos os adolescentes ( $600 \mathrm{UI}$ a adolescentes saudáveis e 1000 UI aos que têm fatores de risco para défice) e o doseamento de 25(OH)D a adolescentes em risco.

O nosso objetivo foi determinar status de vitamina D nos adolescentes utentes do nosso hospital, estimar prevalência de défice e insuficiência e tentar identificar fatores de risco para défice e insuficiência.

\section{METODOLOGIA}

Este estudo decorreu entre janeiro de 2016 e maio de 2017 e entre janeiro e dezembro de 2018. Procedeu-se a determinação dos níveis sérios de $25(\mathrm{OH}) \mathrm{D}$ em adolescentes entre 10 e 18 anos de idade, utentes da consulta externa do Hospital CUF Descobertas e aplicou-se um questionário que avalia variáveis de caracterização demográfica, dados antropométricos, fotótipo, estádio de desenvolvimento pubertário, variáveis relacionadas com hábitos de vida que podem interferir com status da vitamina $D$ nomeadamente, tipo de dieta, exposição solar, utilização de protetor solar, utilização de chapéu, meio de transporte de e para escola, prática desportiva e doenças associadas. Este questionário foi elaborado pelos autores deste trabalho.

Apenas foram admitidos no estudo adolescentes com determinação de 25(OH)D efetuada no laboratório do nosso hospital para garantirmos a mesma técnica laboratorial.

Procedeu-se a análise estatística descritiva da amostra e procurou-se relação estatística entre as diferentes variáveis e os valores de 25(OH)D. A análise estatística foi elaborada utilizando o programa "R", com recurso a teste Chi-squared ou teste Mann-Whitney e Kruskal-wallis com um nível de significância de 5\%.

Considerou-se défice de 25(OH)D valores inferiores a 20 e insuficiência valores inferiores a 30, de acordo com recomendações SAHM.

\section{RESULTADOS}

Foram incluídos neste estudo 320 adolescentes, a maioria do género feminino $(51,6 \%$ - 165). A grande maioria dos adolescentes $(96,6 \%$ - 309) residia em Lisboa e Vale do Tejo. As idades estiveram compreendidas entre 10 e 18 anos, com uma média de 13,6 \pm 2,1 anos. A maioria dos adolescentes (54,0\% - 173) estava na fase média da adolescência, 35,6\% (113) na fase precoce e 10,3\% (34) na fase tardia da adolescência.

À data da consulta, o valor do índice de massa corporal (IMC) variou entre 14,1 - 34,8 kg/m² (média de 20,6 \pm $3,6 \mathrm{~kg} / \mathrm{m}^{2}$ ). A média do valor do IMC foi idêntica em ambos os géneros (20,6 no género masculino e 20,5 no feminino). A classificação em baixo peso, excesso de peso e obesidade foi semelhante em ambos os géneros.

Os doseamentos séricos de vitamina D foram realizados ao longo dos quatro trimestres: $32,9 \%$ no quarto, $27,3 \%$ no terceiro, $21,9 \%$ no primeiro e $17,9 \%$ no segundo trimestre. Os valores de vitamina D variaram entre 6,4 e $61,4 \mathrm{ng} / \mathrm{mL}$, com média de $26,4 \pm 8,5 \mathrm{ng} / \mathrm{mL}$ e foram considerados ótimos em 30,3\% dos doseamentos, insuficientes em 49,1\% e deficientes em 20,6\% dos doseamentos.

A média dos valores de vitamina $D$ foi semelhante no género feminino $(25,7 \pm 7,5 \mathrm{ng} / \mathrm{mL})$ e no género masculino (27,0 $\pm 9,3 \mathrm{ng} / \mathrm{mL})$. Contudo, no género feminino $25,2 \%$ das adolescentes apresentaram valores ótimos de vitamina D, 56,1\% apresentaram insuficiência e 18,7\% deficiência de vitamina D, enquanto no género masculino 35,2\% dos adolescentes apresentaram valores ótimos de vitamina D, 42,4\% apresentaram insuficiência e 22,4\% deficiência de vitamina D. Esta diferen- 
Variação de 25-hidroxivitamina D por idades

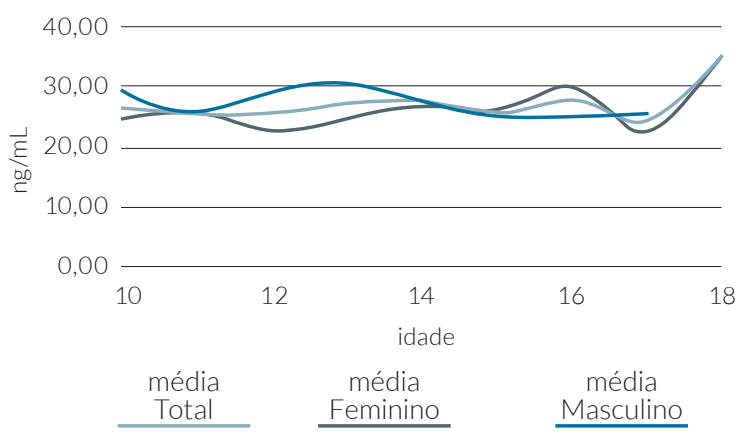

FIGURA 1. Valores vitamina D por idade e género.

TABELA 1. Associação diferentes variáveis com valor de vitamina D.

\begin{tabular}{|c|c|c|}
\hline Variável & $p$-value & Significance \\
\hline Categoria vitamina $D$ versus género & 0,0034 & Yes \\
\hline $\begin{array}{l}\text { Categoria de vitamina D versus fases da } \\
\text { adolescência }\end{array}$ & 0,75 & No \\
\hline $\begin{array}{l}\text { Categoria de vitamina } \mathrm{D} \text { versus } \\
\text { trimestre de análise }\end{array}$ & $<0,001$ & Yes \\
\hline Categoria de vitamina $D$ versus IMC & 0,59 & No \\
\hline Utilização de chapéu por género & 0,54 & No \\
\hline $\begin{array}{l}\text { Utilização de chapéu versus fases de } \\
\text { adolescência }\end{array}$ & 0,96 & No \\
\hline Vitamina $\mathrm{D}$ versus IMC & 0,0161 & Yes \\
\hline $\begin{array}{l}\text { Categoria de vitamina } D \text { versus atividade } \\
\text { desportiva }\end{array}$ & 0,96 & No \\
\hline $\begin{array}{l}\text { Protetor solar versus categoria de } \\
\text { vitamina D }\end{array}$ & 0,21 & No \\
\hline $\begin{array}{l}\text { Atividade desportiva outdoor versus } \\
\text { categoria de vitamina }\end{array}$ & 0,036 & Yes \\
\hline Vitamina $\mathrm{D}$ por trimestre & $<0,001$ & Yes \\
\hline Vitamina D por fase de adolescência & 0,46 & No \\
\hline Vitamina D versus atividade outdoor & 0,22 & No \\
\hline Vitamina D versus utilização de chapéu & 0,8 & No \\
\hline Protetor solar versus vitamina $D$ & & No \\
\hline Vitamina D versus fotótipo & 0,45 & No \\
\hline $\begin{array}{l}\text { Fotótipo versus categoria vitamina D } \\
\text { (agregando o fotótipo IV e V) }\end{array}$ & $<0,001$ & Yes \\
\hline Meio de transporte versus vitamina $\mathrm{D}$ & 0,9 & No \\
\hline
\end{tabular}

ça entre os géneros foi estatisticamente significativa $(p=0,0034)$. A média dos valores de vitamina $D$ foi idêntica nas diferentes fases de adolescência: 25,9 \pm $7,7 \mathrm{ng} / \mathrm{mL}$ na fase precoce, $26,9 \pm 8,8 \mathrm{ng} / \mathrm{mL}$ na fase intermédia e 25,0 \pm 9,3 ng/mL na fase tardia da adolescência (Fig. 1). Não foi encontrada associação estatisticamente significativa entre valores inferiores de vitamina D e IMC mais elevado. Cerca de 18,5\% dos valores de deficiência, 10\% dos valores de insuficiência e 9\% dos valores ótimos de vitamina D corresponderam a adolescentes obesos. Apesar da média dos valores de vitamina D nos adolescentes obesos (23,2 ng/mL no género feminino e $23,4 \mathrm{ng} / \mathrm{mL}$ no género masculino) ser inferior à dos adolescentes normoponderais $(25,4 \mathrm{ng} / \mathrm{mL}$ no género feminino e $27,7 \mathrm{ng} / \mathrm{mL}$ no género masculino), a diferença não foi estatisticamente significativa $(p=0,12)$.

As categorias de valores de vitamina D obtidas por trimestre foram diferentes, a maioria dos valores ótimos de vitamina D foram no terceiro trimestre (58,8\%), insuficiência foi mais frequente no quarto trimestre (39,7\%) e deficiência mais frequente no primeiro trimestre (40,9\%). Esta diferença foi estatisticamente significativa $(p<0,001)$.

Foi encontrada associação entre a categoria dos níveis séricos de vitamina $\mathrm{D}$ e a prática de atividade desportiva outdoor, a maioria dos níveis ótimos (67,7\%) foram encontrados em adolescentes com prática de atividade outdoor e a maioria dos níveis de deficiência (60\%) foram encontrados em adolescentes sem prática desportiva outdoor. Esta diferença foi estatisticamente significativa $(p=0,036)$. Não se verificou diferença entre os géneros quanto à prática desportiva outdoor.

Dos 320 adolescentes, 69 foram classificados quanto ao fotótipo: 17 com fotótipo II, 17 com fotótipo III, 28 com fotótipo IV e 7 adolescentes com fotótipo $V$. A média dos valores de vitamina $D$ foi inferior nos adolescentes com fotótipo IV (24,8 ng/mL) e fotótipo V $(23,9 \mathrm{ng} / \mathrm{mL})$ relativamente aos adolescentes com fotótipo II $(25,4)$ e fotótipo III (26,9), esta diferença foi estatisticamente significativa se considerarmos os fotótipos IV e $\vee$ em conjunto $(p<0,001)$.

Não se encontrou qualquer associação com as outras variáveis estudadas (tipo de dieta, exposição solar, utilização de protetor solar, utilização de chapéu, meio de transporte de e para escola e doenças associadas), provavelmente devido ao baixo número de respostas obtidas às questões relacionadas (Tabela 1).

\section{DISCUSSÃO}

A amostra global deste estudo é significativa ( $n=320$ ), e vem ajudar a melhor compreender a realidade acerca desta vitamina nos adolescentes portugueses. Infelizmente os questionários tiveram várias respostas omissas, o que levou a " $n$ " mais pequenos em algumas variáveis, limitando a capacidade de estabelecer relação estatística entre valores de vitamina D e variáveis estudadas. 
Este trabalho vem mostrar uma prevalência de insuficiência e de défice, de acordo com critérios referidos SAHM, muito elevada, cerca de $50 \%$ e de $20 \%$, respetivamente. Apenas 30\% dos adolescentes apresentaram valores ótimos de $25(\mathrm{OH})$ D. Esta prevalência de $70 \%$ de valores subótimos é extremamente elevada quando comparada com literatura existente.

O valor médio de $25(\mathrm{OH}) \mathrm{D}$ foi de $26,4 \mathrm{ng} / \mathrm{mL}$, o que foi superior ao encontrado em outros estudos portugueses com adolescentes. ${ }^{16,17}$ Este valor é relativamente constante ao longo de toda a adolescência, não se verificando diferenças significativas nas diferentes fases da adolescência.

Quando olhamos para a diferença entre género masculino e feminino verificamos uma média de $25(\mathrm{OH})$ D superior no feminino $(25,5$ vs 27,1$)$ e existe associação estatisticamente significativa entre o género e a prevalência de défice e insuficiência, sendo maior o risco de valores subótimos nas raparigas.

Tal como esperado, e descrito na literatura, a vitamina D tem uma variação sazonal muito clara e foi possível demonstrar, com significado estatístico, valores mais baixos de 25(OH)D nos meses de inverno e maior incidência de défice e insuficiência.

No nosso estudo conseguimos demonstrar de forma significativa que indivíduos com fotótipos IV ou $\vee$ têm maior incidência de valores subótimos de $25(\mathrm{OH}) 25$ que indivíduos dos restantes fotótipos.

A atividade física outdoor parece ser protetora contra o défice desta vitamina.

Não foi possível neste trabalho identificar outros fatores de risco, mas o elevado número de falta de resposta nos questionários impossibilita que se tire conclusões quanto às restantes variáveis estudadas.

Ao verificarmos prevalência de insuficiência de vitamina D tão elevada, coloca-se a dúvida, já levantada na literatura, de qual o cut-off que devemos utilizar ao avaliar valores séricos de $25(\mathrm{OH}) \mathrm{D}$ em adolescentes. A média de $25(\mathrm{OH}) \mathrm{D}$ da nossa amostra encontra-se na faixa da normalidade de acordo com os critérios da Pediatric Endocrine Society.

É fundamental identificar quais os riscos e consequências da insuficiência desta vitamina e quais os valores ideais por forma à formulação de recomendações realistas.

Outra dúvida que se levanta relaciona-se com a variação sazonal desta vitamina. Sabendo-se que existe esta variação na nossa realidade, em que época do ano faz sentido dosear 25(OH)D - no Inverno por forma a determinar o vale ou no Verão por forma a determinar o pico de cada indivíduo.

É importante definir qual a melhor estratégia para cada população:

- Suplementar adolescentes de forma universal, tendo em conta prevalência tão elevada de níveis subótimos e sua baixa toxicidade;

- Suplementar indivíduos com fatores de risco definidos;

- Determinar níveis séricos de 25(OH)D em todos os indivíduos ou apenas nos que têm fatores de risco.

\section{CONCLUSÃO}

A amostra de adolescentes utilizadores da consulta externa do Hospital CUF Descobertas apresenta elevada prevalência de défice e insuficiência de vitamina $D$, sendo que estes valores reforçam a necessidade de rever quais os valores de referência a utilizar nesta faixa etária. O sexo feminino e os fotótipos IV e V são fatores de risco para valores inferiores ao recomendado e a prática de atividade física ao ar livre é fator protetor.

\section{AGRADECIMENTOS / ACKNOWLEDGMENTS}

Os autores agradecem aos adolescentes e famílias que amavelmente colaboraram neste trabalho.

\section{RESPONSABILIDADES ÉTICAS}

CONFLITOS DE INTERESSE: Os autores declaram a inexistência de conflitos de interesse na realização do presente trabalho.

FONTES DE FINANCIAMENTO: Não existiram fontes externas de financiamento para a realização deste artigo.

CONFIDENCIALIDADE DOS DADOS: Os autores declaram ter seguido os protocolos da sua instituição acerca da publicação dos dados de doentes.

PROTEÇÃO DE PESSOAS E ANIMAIS: Os autores declaram que os procedimentos seguidos estavam de acordo com os regulamentos estabelecidos pelos responsáveis da Comissão de Investigação Clínica e Ética e de acordo com a Declaração de Helsínquia da Associação Médica Mundial.

PROVENIÊNCIA E REVISÃO POR PARES: Não comissionado; revisão externa por pares. 


\section{ETHICAL DISCLOSURES}

CONFLICTS OF INTEREST: The authors have no conflicts of interest to declare.

FINANCING SUPPORT: This work has not received any contribution, grant or scholarship

CONFIDENTIALITY OF DATA: The authors declare that they have followed the protocols of their work center on the publication of data from patients.

PROTECTION OF HUMAN AND ANIMAL SUBJECTS: The authors declare that the procedures followed were in accordance with the regulations of the relevant clinical research ethics committee and with those of the Code of Ethics of the World Medical Association (Declaration of Helsinki).

PROVENANCE AND PEER REVIEW: Not commissioned; externally peer reviewed.

\section{REFERÊNCIAS}

1. Ladhani S, Srinivasan L, Buchanan C, Allgrove J. Presentation of vitamin D deficiency. Arch Dis Child. 2004;89:781-784.

2. Najada AS, Habashneh MS, Khader M. The frequency of nutritional rickets among hospitalized infants and its relation to respiratory diseases. J Trop Pediatr. 2004;50:364-368.

3. Pawley NJ, Bishop N. Prenatal and infant predictors of bone health: the influence of vitamin D. Am J Clin Nutr. 2004;80:1748S-1751S.

4. Hatun S, Ozkan B, Orbak Z, et al. Vitamin D deficiency in early infancy. J Nutr. 2005;135:279-82.

5. Misra M, Pacaud D, Petryk A, Collett-Solberg PF, Kappy M, on behalf of the Drug and Therapeutics Committee of the Lawson Wilkins Pediatric Endocrine Society. Vitamin D deficiency in children and its management: review of current knowledge and recommendations. Pediatrics. 2008;122:398-417.

6. Pérez-Lopez FR, Pérez-Roncero G, Lopez-Baena MT. Vitamin D and adolescent health. Adolesc Health Med Ther. 2010;1:18. doi: 10.2147/AHMT.S7472.

7. Misra M. Vitamin D insufficiency and deficiency in children and adolescents. UpToDate 2018. [consultado 2019-0516] Disponível em: https://www.uptodate.com. Acesso em: 16/05/2019.

8. Lehtonen-Veromaa MK, Möttönen TT, Nuotio IO, Irjala KM, Leino AE, Viikari JS. Vitamin D and attainment of peak bone mass among peripubertal Finnish girls: A 3-y prospective study. Am J Clin Nutr. 2002;76:1446e53.

9. Viljakainen HT, Natri AM, Kärkkäinen M, Huttunen MM, Palssa A, Jakobsen J, et al. A positive dose-response effect of vitamin D supplementation on site-specific bone mineral augmentation in adolescent girls: A double-blinded randomized placebo- controlled 1-year intervention. J Bone Miner Res. 2006;21:836e44. doi: 10.1359/jbmr.060302.

10. Winzenberg T, Powel S, Shaw KA, Graeme J. Effects of vitamin D supple- mentation on bone density in healthy children: Systematic review and meta-analysis. BMJ. 2011;342:c7254e65. doi: 10.1136/bmj.c7254.

11. MatsuokaL Y, Wortsman J, Hollis BW.Useoftopicalsunscreenfortheevalu- ation of regional synthesis of vitamin D3. J Am Acad Dermatol. 1990;22:772e5.
12. Terushkin V, Bender A, Psaty EL, Engelsen O, Wang SQ, Halpern AC. et al. Estimated equivalency of vitamin D production from natural sun exposure versus oral vitamin D supplementation across seasons at two US latitudes. J Am Acad Dermatol. 2010;62: 929.e1e9. doi: 10.1016/j.jaad.2009.07.028.

13. Weaver CM, Fleet JC. Vitamin D requirements: Current and future. Am J Clin Nutr. 2004;80:S1735e9.

14. Mansbach JM, Ginde AA, Camargo CA Jr. Serum 25-hydroxyvitamin D levels among US children aged 1 to 11 years: do children need more vitamin D? Pediatrics. 2009; 124:1404-10. doi: 10.1542/peds.2008-2041.

15. Saintonge S, Bang H, Gerber LM. Implications of a new definition of vitamin D deficiency in a multiracial US adolescent population: the National Health and Nutrition Examination Survey III. Pediatrics. 2009; 123:797.

16. Gordon CM, Feldman HA, Sinclair L, Williams AL, Kleinman PK, Perez-Rossello J, et al. Prevalence of vitamin D deficiency among healthy infants and toddlers. Arch Pediatr Adolesc Med. 2008; 162:505-12. doi: 10.1001/archpedi.162.6.505.

17. Cabral M, Araújo J, Lopes C, Barros H, Guimarães JT, Severo $\mathrm{M}$, et al. Relationship between dietary vitamin $\mathrm{D}$ and serum 25-hydroxyvitamin D levels in Portuguese adolescents. Public Health Nutr. 2017: 21: 325-32. doi: 10.1017/ S1368980017002804.

18. Cabral M, Araújo J, Teixeira J, Barros H, Martins S, Guimarães $\mathrm{JT}$, et al. Vitamin D levels and cardiometabolic risk factors in Portuguese adolescents. Int J Cardiol. 2016;220:501-7. doi: 10.1016/j.ijcard.2016.06.154.

19. Willer CL, Dyment DA, Sadovnick AD, Rothwell PM, Murray TJ, Ebers GC; Canadian Collaborative Study Group. Timing of birth and risk of multiple sclerosis: population based study. BMJ. 2005; 330:120. doi: 10.1136/bmj.38301.686030.63.

20. Willer CL, Dyment DA, Sadovnick AD, Lewy H, Ashkenazi I, Laron Z. Seasonality of birth and onset of clinical disease in children and adolescent (0-19 years) with type 1 diabetes mellitus in Canterbury, New Zealand. J Pediatr Endocrinol Metab. 2002; 15:645-7. doi: 10.1515/jpem.2002.15.5.645.

21. Merlino LA, Curtis J, Mikiuls TR, Cerhan JR, Criswell LA, Saag KG; lowa Women's Health Study. Vitamin D intake is inversely associated with rheumatoid arthritis: results from the lowa Women's Health Study. Arthritis Rheum. 2004; 50:72-7. doi: 10.1002/art.11434.

22. Cantorna MT, Munsick C, Bemiss C, Mahon BD. 1,25-Dihydroxycholecalciferol prevents and ameliorates symptoms of experimental murine inflammatory bowel disease. J Nutr. 2000; 130:2648.

23. Mersch PP, Middendorp HM, Bouhuys AL, Beersma DG, van den Hoofdakker RH. Seasonal affective disorder and latitude: a review of the literature. J Affect Discord. 1999; 53:35-48. doi: 10.1016/s0165-0327(98)00097-4.

24. Rasanen P, Hakko H, Jarvelin MR. Prenatal and perinatal risk factors for psychiatric diseases of early onset. Results are different if seasons are ategorised differently. BMJ. 1999; 318:1622.

25. Juonala M, Voipio A, Pahakala K, Viikari JS, Mikkilä V, Kähönen $\mathrm{M}$, et al. Childhood 25-OH vitamin D levels and carotid intima-media thickness in adulthood: the cardiovascular risk in young Finns study. J Clin Endocrinol Metal. 2015; 100:1469-76. doi: 10.1210/jc.2014-3944.

26. Bodiwala D, Luscombe CJ, French ME, Liu S, Saxby MF, Jones PW, et al. Susceptibility to prostate cancer: studies on interactions between UVR exposure and skin type. Carcinogenesis. 2003; 24:711-7. doi: 10.1093/carcin/bgg021. 
27. Garland CF, Comstock GW, garland FC,Helsing KJ, Shaw EK, Gorham ED. Serum 25-OH vitamin D and colon cancer: eight year prospective study. Lancet. 1989;2:1176-8. doi: 10.1016/ s0140-6736(89)91789-3.

28. Garland FC, Garland CF, Gorham ED, Young JF. Geographic variation in breast cancer mortality in the United States: a hypothesis involving exposure to solar radiations. Prev Med. 1990; 19:614.

29. Grant WB. An ecologic study of dietary and solar ultraviolet-B links to breast carcinoma mortality rates. Cancer. 2002;94:272-81. doi: 10.1002/cncr.10196.

30. Pritchard RS, Baron JA, Gerhardsson de Verdier M. Dietary calcium, vitamin D, and the risk of colorectal cancer in Stockholm, Sweden. Cancer Epidemiol Biomarkers Prev. 1996;5:897-900

31. The Society for Adolescent Health and Medicine. Recommended Vitamin D Intake and Management of Low Vitamin D Status in Adolescents: A Position Statement of the Society for Adolescent Health and Medicine. J Adolesc Health. 2013;52:801-3. doi: 10.1016/j.jadohealth.2013.03.022.

32. Misra M, Pacaud D, Petryk A, Collett-Solberg PF, Kappy M; Drug and Therapeutics Committee of the Lawson Wilkins Pediatric Endocrine Society. Vitamin D deficiency in children and its management: review of current knowledge and recommendations. Pediatrics. 2008; 122:398-417. doi: 10.1542/ peds.2007-1894.

33. Peroni DG, Trambusti I, Di Cicco ME, Nuzzi G. Vitamin D in pediatric health and disease. Pediatr Allergy Immunol. 2020 ;31 Suppl 24:54-57. doi: 10.1111/pai.13154. 